\title{
Author Correction: Prior shared success predicts victory in team competitions
}

Satyam Mukherjee (D), Yun Huang, Julia Neidhardt (D), Brian Uzzi and Noshir Contractor

Correction to: Nature Human Behaviour https://doi.org/10.1038/s41562-018-0460-y, published online 3 December 2018.

In the version of this article initially published, errors occurred in the Acknowledgments. The correct text is as follows:

This research was funded by grants from the Northwestern University Clinical and Translational Sciences Institute (NUCATS), the Northwestern University Institute for Complex Systems (NICO), the National Institutes of Health (1R01GM112938-01), the US Army Research Laboratory and US Army Research Office Grant W911NF-15-1-0577, the Army Research Laboratory (grant W911NF-09-2-0053), and the Army Research Office (grant W911NF-14-10686). The funders had no role in study design, data collection and analysis, decision to publish or preparation of the manuscript.

The error has been corrected in the HTML and PDF versions of the article.

Published online: 13 March 2019

https://doi.org/10.1038/s41562-019-0581-y 